\title{
Weak+Vacuum and One Decoy States with Two Way Quantum Key Distribution Protocol
}

\author{
M. F. Abdul Khir ${ }^{1,2}$, Iskandar Bahari ${ }^{3}$, S. Ali $^{4}$, S. Shaari ${ }^{1}$ \\ ${ }^{1}$ Photonics Lab, Institute of Micro Engineering and Nanoelectronics (IMEN), \\ Universiti Kebangsaan Malaysia, 43400 UKM Bangi, Malaysia. \\ ${ }^{2}$ Photonics Lab, Photonic Technology \& Product Development, \\ ${ }^{3}$ Advance Analysis and Modelling, \\ Mimos Berhad, Technology Park Malaysia, 57000 Kuala Lumpur, Malaysia \\ ${ }^{4}$ Department of Science in Engineering, Faculty of Engineering, \\ International Islamic University of Malaysia (IIUM), Jalan Gombak, 53100 Kuala \\ Lumpur, Malaysia
}

\begin{abstract}
We present relevant bounds for the case of weak+vacuum decoy state and one decoy state for a two way four states Quantum Key Distribution (QKD) protocol. The numerical simulation result was significant given that an improvement in maximum secure distance of nearly double is achieved.
\end{abstract}

\section{INTRODUCTION}

Since the introduction of the first Quantum Key Distribution (QKD) protocol, namely BB84 by Bennet and Brassard in 1984 [1], rapid progress in the field has been recorded, from theoretical down to experimental aspects [2]. In recent progresses, attempts of applying QKD into classical network infrastructure were also reported [3-6]. With its security guaranteed through law of physics, QKD has managed to provide the unconditionally secure method for secret key sharing between two distant parties. However, with the absent of ideal equipments, realization of an information theoretically secure QKD appeared less practical. The unavailability of true single photon source has made most QKD systems rely on weak laser pulses which inevitably emit multi photon pulses, a situation that invites powerful attacks such as Photon Number Splitting (PNS) attack. While the security of the shared key remains unconditionally secure, this limitation severely affects the secure key generation rate and the maximum secure distance.

Fortunately, with recent introduction of decoy state method by Hwang [7] and further development by such as in [8 14], the weak laser pulses based QKD system is made practical again. Since then, applications of decoy state have been demonstrated successfully in several experimental works [15 18]. Recently, progresses in decoy states with regards to QKD protocols other than BB84 have also been reported such as found in the work by $[19,20]$ for SARG04. For two way QKD protocols [21 29], practical decoy state extension can be seen in the work of 
Shaari et al in [30]. Their result with two decoy states for the LM05 protocol was encouraging given that the secure distance has now been extended by almost double.

In our previous work in [32], we have considered the first approach suggested in [30] where the single and double photon contributions were separately calculated, to accommodate the case of weak+vacuum decoy state. In this work, we simplify the bound in the second approach in [30] where the single and double photon contributions were lumped and also the case for one decoy state with both approaches. We then conduct numerical analysis of the proposed bounds. In order to find out how well the proposed scheme would perform, we also compare against the case of without decoy state and the case of infinite decoy state. As such, this letter is organized as follows. Section two describes the proposed scheme. Section three discusses the results and section four conclude and suggest future works.

\section{DECOY STATE ESTIMATIONS}

\subsection{Infinite Decoy State}

Before we proceed with the two cases of weak+vacuum and one decoy states, let us first review the case of theoretical limit in which we assume that we have an infinite number of decoy states. We assume a two way QKD system with channel transmission $t_{C}=10^{-\left(\frac{2 \alpha l_{c}}{10}\right)}$ where $\alpha$ is the optical fiber loss coefficient and $l_{c}$ is the transmission distance. Notice the factor of two which represents the two way quantum channel of this two way protocol in which we assume the same loss at both forward and backward channel. The overall transmission and detection efficiency is then $\eta=t_{C} \eta_{B o b}$ and the transmittance of $\mathrm{i}$-th photon state $\eta_{i}=1-(1-\eta)^{i}$. The yield $Y_{i}$, gain $Q_{i}$ and error rate $E_{i}$ for i-th photon states is given in [9] as :

$$
\begin{gathered}
Y_{i}=Y_{0}+\eta_{i}-\eta Y_{0} \cong Y_{0}+\eta_{i} \\
Q_{i}=Y_{i} \frac{\mu^{i}}{i !} e^{-\mu} \\
E_{i}=\frac{e_{0} Y_{0}+e_{\text {detector }} \eta_{i}}{Q_{i}}
\end{gathered}
$$

where $Y_{0}$ is background detection events, $e_{0}$ is noise error rate, assumed as $1 / 2$ due to randomness and $e_{\text {detector }}$ is the erroneous signal detection probability.

The overall Signal Gain $\left(Q_{\mu}\right)$ and Quantum Bit Error Rate QBER $\left(E_{\mu}\right)$ as given in [9] is then

$$
Q_{\mu}=\sum_{i=0}^{\infty} Y_{i} \frac{\mu^{i}}{i !} e^{-\mu}=Y_{0}+1-e^{-\eta \mu}
$$




$$
E_{\mu}=\frac{e_{0} Y_{0}+e_{\text {detector }}\left(1-e^{-\eta \mu}\right)}{Q_{\mu}}
$$

\subsection{Weak+Vacuum Decoy State}

The weak+vacuum decoy state was first proposed by Lo et al as the optimal case for a practical two decoy states with one as a vacuum state and the other as a weak state [9]. The key advantage in the weak+vacuum decoy state is that Bob and Alice can estimate the background rate correctly through the vacuum decoy state [9]. This then lead to a better bound and was in fact shown to be optimal for BB84 in [9].

In the course of extending decoy state into a two way protocol, one should also consider the gain from double photon pulses besides the single photon pulses. This was the major consideration in the work of [30] which results into two key rate formulas. While the first, represented in Eq 25 of [30] calculates single and double photon contributions separately, the second, represented in Eq 26 lumped both contributions. We describe the case for the former which is adapted from our previous work in [32] in sub-section A and the latter in sub-section B.

\section{A. The case for $R_{1+2}$}

As previously mentioned, in this case, contribution from single and double photon pulses are calculated separately. For single photon contribution, we can directly use the one suggested by Ma et al in [9] while for double photon contribution we need to derive from the one suggested for the case of two decoy state by Shaari et al [30]. As such, the lower bound of the yield and gain of single photon state denoted respectively as $\left(Y_{1}\right)$ and $\left(Q_{1}\right)$ in [9], are given as :

$$
\begin{gathered}
Y_{1} \geq Y_{1}^{L}=\frac{\mu}{\mu v-v^{2}}\left(Q_{v} e^{v}-Q_{\mu} e^{\mu} \frac{v^{2}}{\mu^{2}}-\frac{\mu^{2}-v^{2}}{\mu^{2}} Y_{0}\right) \\
Q_{1} \geq Q_{1}^{L}=\frac{\mu^{2} e^{-\mu}}{\mu \nu-v^{2}}\left(Q_{v} e^{v}-Q_{\mu} e^{\mu} \frac{v^{2}}{\mu^{2}}-\frac{\mu^{2}-v^{2}}{\mu^{2}} Y_{0}\right)
\end{gathered}
$$

Similar to the derivation of Weak+Vacum decoy state in [9], taking $v_{2}=0$ and replacing gain from second decoy state $Q_{v 2}$ with $Y_{0}$ and its corresponding error rate with $e_{0}$ in Eq 10 and 11 of [30] we obtain the lower bound of double photon yield $\left(Y_{2}^{L}\right)$ and gain $\left(Q_{2}^{L}\right)$ for the case of Weak+Vacum decoy state as :

$$
Y_{2} \geq Y_{2}^{L}=\frac{2 \mu\left(Q_{v} e^{v}-\frac{v^{3}}{\mu^{3}} Q_{\mu} e^{\mu}-\frac{\mu^{3}-v^{3}}{\mu^{3}} Y_{0}-\frac{v \mu^{2}-v^{3}}{\mu^{2}} Y_{1}^{U}\right)}{v^{2} \mu-v^{3}}
$$




$$
Q_{2} \geq Q_{2}^{L}=\frac{\mu^{3} e^{-\mu}\left(Q_{v} e^{v}-\frac{v^{3}}{\mu^{3}} Q_{\mu} e^{\mu}-\frac{\mu^{3}-v^{3}}{\mu^{3}} Y_{0}-\frac{v \mu^{2}-v^{3}}{\mu^{2}} Y_{1}^{U}\right)}{v^{2} \mu-v^{3}}
$$

where $Y_{1}^{U}$ is given by :

$$
Y_{1}^{U}=\frac{\left(2 Q_{v} e^{v}-2 Y_{0}-Y_{2}^{\infty} v^{2}\right)}{2 v}
$$

Note that the $Y_{2}^{\infty}$ is the double photon yield from the case of infinite decoy state.

Doing the same for Eq 15 and 16 of [30], the upper bound of single and double photon error rate denoted as $e_{1}^{U}$ and $e_{2}^{U}$ respectively is then given as :

$$
\begin{aligned}
& e_{1} \leq e_{1}^{U}=\frac{E_{v} Q_{v} e^{v} \mu^{2}-E_{\mu} Q_{\mu} e^{\mu} v^{2}-e_{0} Y_{0}\left(\mu^{2}-v^{2}\right)}{Y_{1}^{L}\left(v \mu^{2}-\mu v^{2}\right)} \\
& e_{2} \leq e_{2}^{U}=\frac{2\left(E_{v} Q_{v} e^{v} \mu-E_{\mu} Q_{\mu} e^{\mu} v-e_{0} Y_{0}(\mu-v)\right)}{Y_{2}^{L}\left(\mu v^{2}-v \mu^{2}\right)}
\end{aligned}
$$

The resulted $Y_{2}, Q_{2}, e_{1}$ and $e_{2}$ together with $Y_{1}$ and $Q_{1}$ can now directly be plugged into the secure key rate formula in Eq 28.

\section{B. The case for $R_{12}$}

Similar to the case of $R_{1+2}$, taking $v_{2}=0$ and substituting the gain from the second decoy state $Q_{v 2}$ with $Y_{0}$ and its corresponding error rate with $e_{0}$, we rewrite the lower bound $(Y 1+Y 2)^{L}$ in Eq. 19 of [30] for the case of "weak+vacuum", which is now given as :

$$
(Y 1+Y 2)^{L}=\frac{\mu^{3} e^{v} Q_{v}-\left(\mu^{3}-v^{3}\right) Y_{0}-v^{3} Q_{\mu} e^{\mu}+\left(v^{3} \mu-\frac{1}{2} v^{3} \mu^{2}\right) Y_{1}^{L}}{\mu^{3}\left(v-\frac{1}{2} \frac{v^{3}}{\mu}\right)}
$$

where $Y_{1}^{L}$ is from Eq. 7.

The lower bound of effective gain $Q_{12}^{L}(\mu)$ and upper bound of effective error rate $\varepsilon^{U}$ is given by [30] as :

$$
Q_{12}^{L}(\mu)=\left[\frac{(Y 1+Y 2)^{L}}{2} \mu^{2}+\left(Y_{1}^{L} \mu-\frac{Y_{1}^{L} \mu^{2}}{2}\right)\right] e^{-\mu}
$$




$$
{ }_{\varepsilon}^{U}=\frac{E_{\mu} Q_{\mu}-e_{0} Y_{0} e^{-\mu}}{Q_{12}^{L}}
$$

The effective gain $\left(Q_{12}^{L}(\mu)\right)$ and error rate $\left(\varepsilon^{U}\right)$ can be plugged into the following Eq 29 for the lower bound of key generation rate

\subsection{One Decoy State}

The case of one decoy state is similar to the weak+vacuum decoy state except that Bob and Alice do not know their $Y_{0}$ precisely [9]. Hence they have to estimate the upper bound $\left(Y_{0}^{U}\right)$ which can be imported directly from [9] and is given as :

$$
Y_{0} \leq Y_{0}^{U}=\frac{E_{\mu} Q_{\mu} e^{\mu}}{e_{0}}
$$

From here, we describe the case of $R_{1+2}$ in subsection $\mathrm{A}$ and $R_{12}$ in subsection $\mathrm{B}$.

\section{A. One Decoy State using $R_{1+2}$}

Substituting Eq. 16 into Eq. 6 and Eq. 7, we obtain the yield and gain of single photon state denoted as $\left(Y_{1}\right)$ and $\left(Q_{1}\right)$ as :

$$
\begin{aligned}
& Y_{1} \geq Y_{1}^{L}=\frac{\mu}{\mu v-v^{2}}\left(Q_{v} e^{v}-Q_{\mu} e^{\mu} \frac{v^{2}}{\mu^{2}}-E_{\mu} Q_{\mu} e^{\mu} \frac{\mu^{2}-v^{2}}{e_{0} \mu^{2}}\right) \\
& Q_{1} \geq Q_{1}^{L}=\frac{\mu^{2} e^{-\mu}}{\mu v-v^{2}}\left(Q_{v} e^{v}-Q_{\mu} e^{\mu} \frac{v^{2}}{\mu^{2}}-E_{\mu} Q_{\mu} e^{\mu} \frac{\mu^{2}-v^{2}}{e_{0} \mu^{2}}\right)
\end{aligned}
$$

Similarly, replacing Eq. 16 into Eq.8 and Eq.9, we obtain the yield and gain of the double photon state denoted respectively as $\left(Y_{2}\right)$ and $\left(Q_{2}\right)$, as :

$$
\begin{gathered}
Y_{2} \geq Y_{2}^{L}=\frac{2 \mu\left(Q_{v} e^{v}-Q_{\mu} e^{\mu} \frac{v^{3}}{\mu^{3}}-E_{\mu} Q_{\mu} e^{\mu} \frac{\mu^{3}-v^{3}}{e_{0} \mu^{3}}-\frac{v \mu^{2}-v^{3}}{\mu^{2}} Y_{1}^{U}\right)}{v^{2} \mu-v^{3}} \\
Q_{2} \geq Q_{2}^{L}=\frac{\mu^{3} e^{-\mu}\left(Q_{v} e^{v}-Q_{\mu} e^{\mu} \frac{v^{3}}{\mu^{3}}-E_{\mu} Q_{\mu} e^{\mu} \frac{\mu^{3}-v^{3}}{e_{0} \mu^{3}}-\frac{v \mu^{2}-v^{3}}{\mu^{2}} Y_{1}^{U}\right)}{v^{2} \mu-v^{3}}
\end{gathered}
$$

where $Y_{1}^{U}$ is given by :

$$
Y_{1}^{U}=\frac{\left(2 Q_{v} e^{v}-2 Y_{0}^{L}-Y_{2}^{\infty} v^{2}\right)}{2 v}
$$

The $Y_{0}^{L}$ in Eq.20 can be obtained following Eq 18 from [9], given as : 


$$
Y_{0}^{L}=\max \left\{\frac{\left(v_{1} Q_{v 2} e^{v 2}-v_{2} Q_{v 1} e^{v 1}\right)}{v_{1}-v_{2}}, 0\right\}
$$

where if we take $v 2=0$ and $Q_{v 2}=0$, we obtain $Y_{0}^{L}=0$.

The upper bound of single and double photon error rate $e_{1}^{U}$ and $e_{2}^{U}$ is given by :

$$
\begin{aligned}
& e_{1} \leq e_{1}^{U}=\frac{E_{v} Q_{v} e^{v} \mu^{2}-E_{\mu} Q_{\mu} e^{\mu} v^{2}-e_{0} Y_{0}^{L}\left(\mu^{2}-v^{2}\right)}{Y_{1}^{L}\left(v \mu^{2}-\mu v^{2}\right)} \\
& e_{2} \leq e_{2}^{U}=\frac{2\left(E_{v} Q_{v} e^{v} \mu-E_{\mu} Q_{\mu} e^{\mu} v-e_{0} Y_{0}^{L}(\mu-v)\right)}{Y_{2}^{L}\left(\mu v^{2}-v \mu^{2}\right)}
\end{aligned}
$$

The resulted $Y_{2}, Q_{2}, e_{1}$ and $e_{2}$ together with $Y_{1}$ and $Q_{1}$ can now directly be plugged into the key rate formula in Eq 28.

\section{B. One decoy state using $R_{12}$}

Similar to the case of $R_{1+2}$, replacing the upper bound of $Y_{0}^{U}$ in Eq.16 into Eq.13, we obtain the lower bound $(Y 1+Y 2)^{L}$ as :

$$
(Y 1+Y 2)^{L}=\frac{\mu^{3} Q_{v} e^{v}-\left(\mu^{3}-v^{3}\right) \frac{E_{\mu} Q_{\mu} e^{\mu}}{e_{0}}-v^{3} Q_{\mu} e^{\mu}+\left(v^{3} \mu-\frac{1}{2} v^{3} \mu^{2}\right) Y_{1}^{L}}{\mu^{3}\left(v-\frac{1}{2} \frac{v^{3}}{\mu}\right)}
$$

where $Y_{1}^{L}$ is from Eq. 6.

The lower bound of effective gain $Q_{12}^{L}(\mu)$ is now given as :

$$
Q_{12}^{L}(\mu)=\left[\frac{(Y 1+Y 2)^{L}}{2} \mu^{2}+\left(Y_{1}^{L} \mu-\frac{Y_{1}^{L} \mu^{2}}{2}\right)\right] e^{-\mu}
$$

where the $(Y 1+Y 2)^{L}$ and $Y_{1}^{L}$ is from Eq. 25 and Eq.6 respectively.

Taking $Y_{0}=0$ in Eq.15, then the upper bound of effective error rate $\varepsilon^{U}$ is now given as :

$$
\varepsilon^{U}=\frac{E_{\mu} Q_{\mu}}{Q_{12}^{L}}
$$

The effective gain $\left(Q_{12}^{L}(\mu)\right)$ and error rate $\left(\varepsilon^{U}\right)$ can be plugged into the following Eq 29 for the lower bound of key generation rate 


\subsection{The Secure Key Rate}

The lower bound of the secure key rate for the case of $R_{1+2}$ and $R_{12}$ is respectively given by [30] as :

$$
\begin{gathered}
R_{1+2} \geq R_{1+2}^{L}=-Q_{\mu} f\left(E_{\mu}\right) H\left(E_{\mu}\right)+\sum_{i=1}^{2} Q_{i}\left[1-\tau\left(e_{i}\right)\right] \\
R_{12} \geq R_{12}^{L}=-Q_{\mu} f\left(E_{\mu}\right) H\left(E_{\mu}\right)+Q_{12}^{L}\left[1-\tau\left(\varepsilon^{U}\right)\right]
\end{gathered}
$$

where

$H\left(E_{\mu}\right)$ is the binary Shannon Entrophy and is given by

$$
H\left(E_{\mu}\right)=-E_{\mu} \log _{2}\left(E_{\mu}\right)-\left(1-E_{\mu}\right) \log _{2}\left(1-E_{\mu}\right)
$$

and $\tau(e)$ as

$\tau(e)=\log _{2}\left(1+4 e-4 e^{2}\right)$ for $e<\frac{1}{2}$ and $\tau(e)=1$ if $e \geq \frac{1}{2}$

\section{RESULTS AND DISCUSSION}

In our numerical analysis, we have made use of reliable data obtained from GYS experiment [31]. Using $f\left(E_{\mu}\right)$ so as to match with [30] and the value of $Y_{0}, \eta_{B o b}$ and $e_{\text {detector }}$ from [31], we solved optimal $\mu$ and $v$ numerically and obtain maximum secure key rate for every distance until it hits zero. The last distance before secure key rate hits zero is treated as the maximum secure distance. We have also conducted numerical simulation against the case of without decoy state as well as the case of theoretical infinite decoy state as a base comparison to estimate how well the proposed scheme would performs. For the case of without decoy state, we based on the one in [26]. The result from numerical simulation is depicted in FIG 1. 


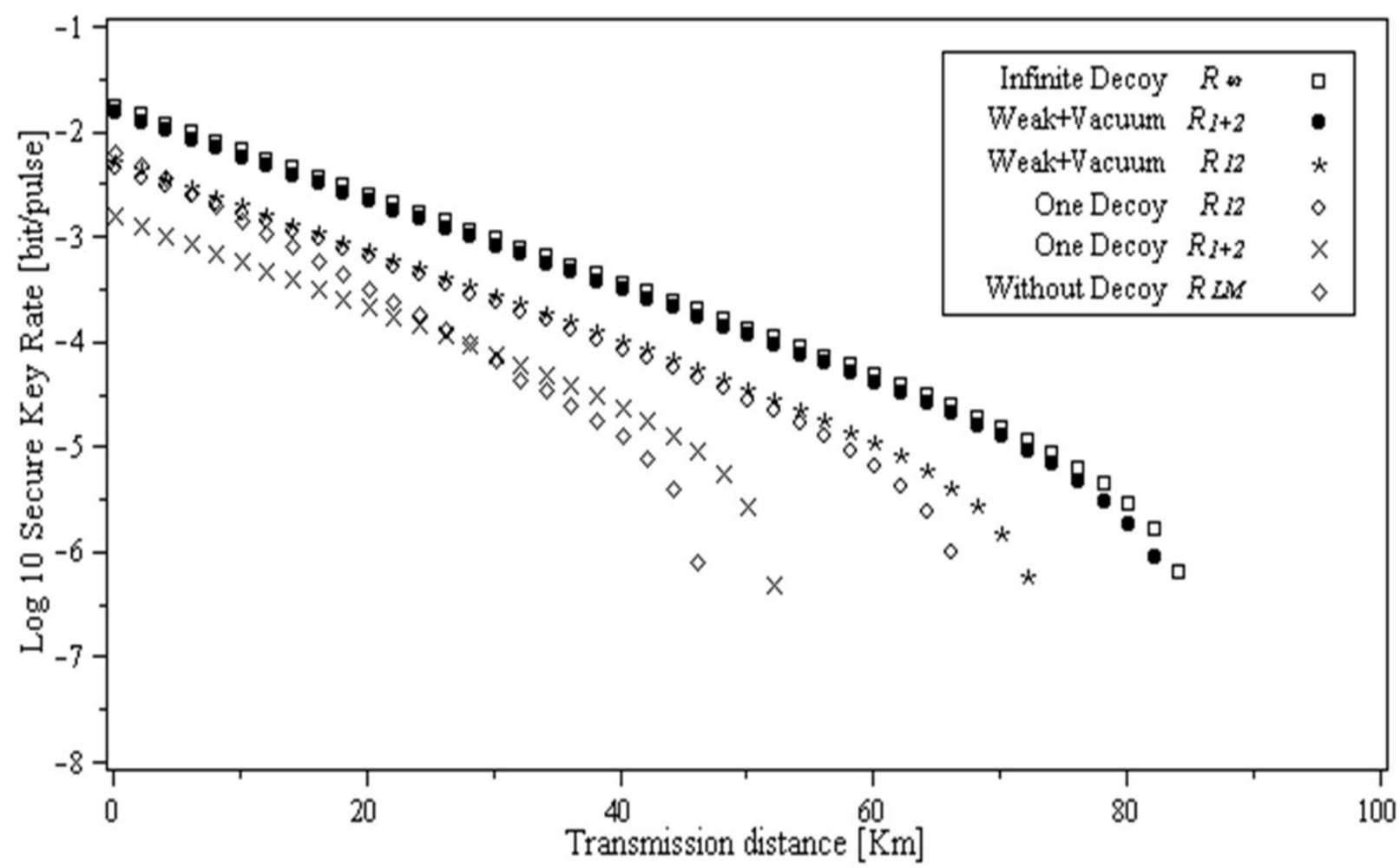

FIG. 1 A plot of key generation rate against transmission distance from the result of numerical simulation for all the six cases mentioned in the text (Infinite decoy state $\left(R_{\infty}\right)$, weak+vacuum decoy state $\left(R_{1+2}\right.$ and $\left.R_{12}\right)$, one decoy state ( derived from $R_{1+2}$ and $\left.R_{12}\right)$ and without decoy state $\left(R_{L M}\right)$. The system parameters are from GYS [31].

It can easily be seen that for the case of $R_{1+2}$, the maximum secure distance is extended by almost double while for the case of $R_{12}$ although not as good as $R_{1+2}$, was able to extend by almost two third the maximum distance of the case of without decoy state. Comparing against the theoretical infinite case, $R_{1+2}$ performs very well close to the limit while $R_{12}$ was a bit shorter by around $10 \mathrm{~km}$. Another aspect of the $R_{12}$ result is that the secure key rate is a bit low with a consistent gap until near to $60 \mathrm{~km}$. The results match well with one in [30] and indicate the practicality of the weak+vacuum decoy state in extending the maximum secure distance of a two way protocol specifically the LM05.

For the case of one decoy state, the one derived for the $R_{12}$ formula had surprisingly performed better than the one derived from the $R_{1+2}$ and was quite close to the case of $R_{12}$ (weak+vacuum). Although the maximum secure distance of one decoy state using $R_{1+2}$ formula was slightly higher than the case of without decoy state, the secure key rate prior to $30 \mathrm{~km}$ was unexpectedly worse. This indicates the impracticality of the proposed bound.

\section{CONCLUSION AND FUTURE WORKS}

We have derived the bounds for specific case of weak+vacum decoy state and one decoy state with a two way Quantum Key Distribution protocol namely the LM05. The numerical simulation results for the weak+vacuum decoy state was quite encouraging given that the maximum secure 
distance was extended by almost double and was very well close to the case of maximum theoretical limit. The result also indicates the practicality of one of the proposed bound for the case of one decoy state that is when the yield calculation of single and double photon contribution was lumped. With all the practical bounds ready, it is very much interesting to see the proposed decoy state extension in action. A simple extension to the source as well as modification of the programming on our previously developed free space based LM05 QKD system in [33] is sufficient enough to accommodate the proposed decoy state extension.

\section{REFERENCES}

[1] C.H. Bennet and G. Brassard," Quantum Cryptography: Public Key Distribution and Coin Tossing”, in Proc of IEEE Int Conference on Computers, systems, and Signal Processing (Bangalore, India, 1984), pp 175179.

[2] N. Gisin, G. Ribordy, W. Tittel and H. Zbinden, “Quantum Cryptography”, Rev. of Mod. Phys. 74, 145 (2002)

[3] Teng-Yun Chen, Jian Wang, Hao Liang, Wei-Yue Liu, Yang Liu, Xiao Jiang, Yuan Wang, Xu Wan, Wei-Qi Cai, Lei Ju, Luo-Kan Chen, Liu-Jun Wang, Yuan Gao, Kai Chen, Cheng-Zhi Peng, Zeng-Bing Chen, and Jian-Wei Pan, "Metropolitan all-pass and inter-city quantum communication network", Optics Express, Vol. 18, Issue 26, pp. 27217-27225 (2010)

[4] M. T. Nguyen-Thi, M. A. Sfaxi, and S. Ghernouti-Hélie, "802.11i Encryption Key Distribution Using Quantum Cryptography," Journal of Network, vol. 1, pp. 9-20, 2006.

[5] Pornsuwanchroen, N., Jamsai, M., Yupapin, P.P., "QKD and QDC via optical-wireless link for quantum mobile telephone network application", International Journal for Light and Electron Optics, 121 (12), 1123-1128 2010

[6] Taengtang, T., Praithoonwattanakit, K., Yupapin, P.P., Suchat, S. "Error corrections of quantum key distribution of the quantum codes via optical wireless link", International Journal for Light and Electron Optics, 122 (5) , 391-394 2011

[7] W. Y. Hwang, "Quantum Key Distribution with High Loss: Toward Global Secure Communication”,Phys. Rev. Lett. 91, 057901 (2003)

[8] Hoi-Kwong Lo, Xiongfeng Ma, and Kai Chen,Phys. Rev.Lett. 94, 230504 (2005).

[9] Xiongfeng Ma,Being Qi,Yi Zhao and Hoi-Kwong Lo, arXiv:quant-ph/0503005v5 10 May 2005.

[10] Xiang-Bin Wang, "Beating the PNS attack in practical quantum cryptography", Phys. Rev. Lett., 94, 230503 (2005) 
[11] J.W. Harrington J.MEttinger, R.J. Hughes, J.E. Nordholt, "Enhancing practical security of quantum key distribution with a few decoy states", quant-ph/0503002

[12] P Rice, J.W. Harrington,"Numerical analysis of decoy state quantum key distribution protocols", arXiv:0901.0013v2

[13] X.-B. Wang, "Decoy-state quantum key distribution with large random errors of light intensity", Phys. Rev. A 75, 052301 (2007)

[14] X.-B.Wang, C.-Z. Peng, J. Zhang, L. Yang and J.-W. Pan, "General theory of decoy-state quantum cryptography with source errors", Phys. Rev. A 77, 042311 (2008)

[15] Y. Zhao, B. Qi, X. Ma, H.-K. Lo, and L. Qian, "Experimental quantum key distribution with decoy states", Physical Review Letters, 96: 070502 (2006).

[16] Y. Zhao, B. Qi, X. Ma, H.-K. Lo, and L. Qian, "Simulation and implementation of decoy state quantum key distribution over $60 \mathrm{~km}$ telecom fiber", in Proceedings of IEEE International Symposium on Information Theory (IEEE, 2006), pp. 2094-2098.

[17] T. Schmitt-Manderbach, H. Weier, M. Fürst, R. Ursin, F. Tiefenbacher, T. Scheidl, J. Perdigues, Z. Sodnik, C. Kurtsiefer, J. G. Rarity, A. Zeilinger, H. Weinfurter, "Experimental Demonstration of Free-Space Decoy state Quantum Key Distribution over 144 km," Phys. Rev.Lett. 98, 010504 (2007).

[18] Y Liu, T.Y. Chen, J. Wang, W. Q. Cai, X. Wan, L. K. Chen, J. H. Wang, S. B. Liu, H. Liang, L. Yang, C. Z. Peng, K. Chen, Z. B. Chen, and J. W. Pan, "Decoy-state quantum key distribution with polarized photons over 200 km", Optics Express, Vol. 18, Issue 8, pp. 8587-8594 (2010)

[19] ShengLi Zhang, XuBo Zou, ChenHui Jin and GuangCan Guo, "Closing the gap of secure quantum key rate with the Heralded Pair-Coherent States", arXiv:0807.1760v1 [quantph] $11 \mathrm{Jul} 2008$

[20] ShengLi Zhang, XuBo Zou , ChuanFeng Li, ChenHui Jin and GuangCan Guo, "Characterization of photon statistics in a single-photon source via variable attenuation", Chinese Science Bulletin 54, 11 (2009)

[21] K. Bostroem, T. Felbinger, "Deterministic Secure Direct Communication Using Entanglement", Phys. Rev. Lett. 89 (2002) 187902

[22] Q.-Y. Cai, B.W. Li, "Deterministic Secure Communication Without Using Entanglement", Chinese Phys. Lett. 21 (2004) 601.

[23] F.-G. Deng, G.L. Long, "Bidirectional quantum key distribution protocol with practical faint laser pulses", Phys. Rev. A 70 (2004) 012311 
[24] M. Lucamarini, S. Mancini, "Secure Deterministic Communication Without Entanglement”, Phys. Rev. Lett. 94 (2005) 140501.

[25] J.S. Shaari, M. Lucamarini, M.R.B. Wahiddin, "Deterministic six states protocol for quantum communication", Physics Letters A, Volume 358, Issue 2,9 October 2006, Pages 85-90

[26] M. Lucamarini, A. Cerè, G. Di Giuseppe, S. Mancini, D. Vitali, and P. Tombesi, "Twoway Protocol for Quantum Cryptography with Imperfect Devices”, Open Syst. \& Inform. Dynam. 14, 169 (2007).

[27] M Ostermeyer, N Walenta, "On the Implementation of a Deterministic Secure Coding Protocol using Polarization Entangled Photons", Optics Communications Volume 281, Issue 17, 1 September 2008, Pages 4540-4544

[28] A. Cere, M. Lucamarini, G. Di Giuseppe, and P. Tombesi, Phys. Rev. Lett. 96, 200501 (2006)

[29] R. Kumar, M. Lucamarini, G. Di Giuseppe, R. Natali, G. Mancini, P. Tombesi, "Twoway quantum key distribution at telecommunication wavelength", Phys. Rev. A 77 022304 (2008)

[30] J.S. Shaari, Iskandar Bahari, Sellami Ali," Decoy states and two way quantum key distribution schemes", Optic Communications, 284 (2011) 697-702

[31] C. Gobby, Z. L. Yuan, and A. J. Shields, Applied Physics Letters, Volume 84, Issue 19, pp. 3762-3764, (2004).

[32] M.F. Abdul Khir, Iskandar Bahari, A Annuar Ehsan, "Two Way Quantum Key Distribution Protocol with Weak+Vacuum Decoy State", accepted for $2^{\text {nd }}$ IEEE International Conference on Photonic (ICP2011), 17 19 October 2011, Kota Kinabalu.

[33] M.F. Abdul Khir, M. N. Mohd Zain, Suryadi, S. Saharudin, S. Shaari, arXiv:quantph/1108.3411v1, 17 Aug 2011 